\section{Surgical management of Launois-Bensaude syndrome}

\author{
E T Brackenbury, W E Morgan
}

\author{
Abstract \\ The presentation, physical findings, and \\ surgical management of a patient with ex- \\ tensive Launois-Bensaude syndrome are \\ reported. \\ (Thorax 1997;52:834-835)
}

Keywords: Madelung's disease, Launois-Bensaude syndrome, lipomatosis.

\section{Case report}

A 53 year old brewery worker (height $1.74 \mathrm{~m}$, weight $103 \mathrm{~kg}$ ) was admitted with a five month history of increasing dyspnoea and unproductive cough. He had no other symptoms of note but admitted to noticing an increase in soft tissue of the neck over the preceding few years. His past medical history was unremarkable and he was on no medication. His alcohol consumption was approximately 40 units per week.

Examination revealed an obese man with abnormally excessive distribution of fatty tissue around the shoulder girdle (fig 1). There was

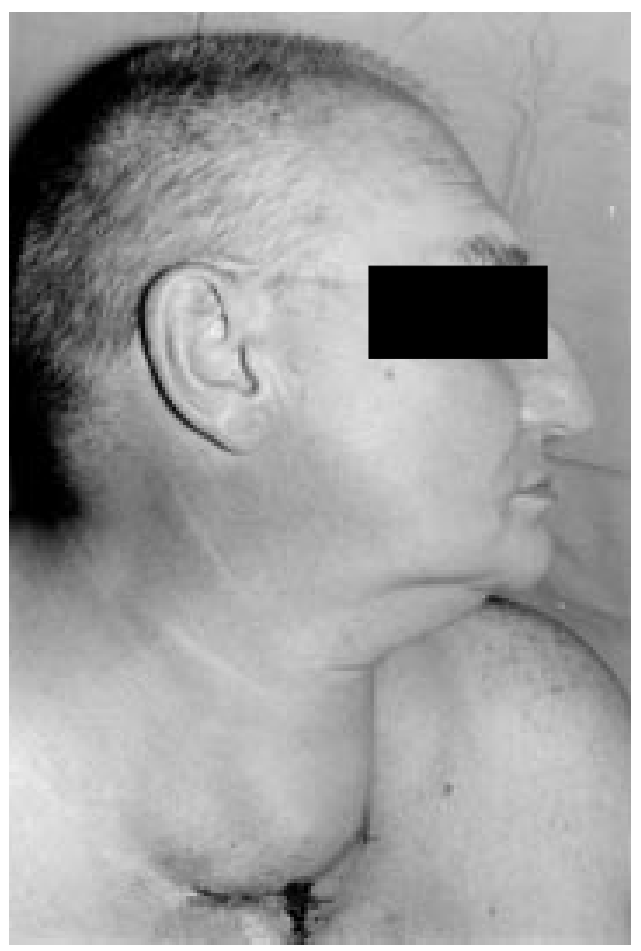

Figure 1 Clinical postoperative photograph of patient showing abnormal distribution of fat around neck and shoulder girdle. markedly decreased air entry on the right hemithorax which was dull to percussion.

Chest radiography showed dense opacification of the right hemithorax suggestive of right upper lobe collapse. Routine full blood count, urea, electrolytes, and liver enzymes were within normal limits except for a raised concentration of lactic dehydrogenase $(\mathrm{LDH})$ of $747 \mathrm{U} / 1$ (normal range $=230-460$ ). The creatinine phosphokinase (CPK) concentration was also raised at $603 \mathrm{U} / 1$ (normal range $=$ 25-195). In view of the raised LDH further tumour markers for germ cell malignancy were measured and $\alpha$-fetoprotein and $\beta$-human chorionic gonadotropin levels were found to be within normal limits.

Computed axial tomographic scanning demonstrated a large mass in the anterior mediastinum, possibly a teratoma. There was also a congenital abnormality at the first right costosternal junction (fig 2) which showed as the only area of increased uptake on isotopic bone scanning.

Spirometric testing revealed a restrictive picture with forced expiratory volume in one second $\left(\mathrm{FEV}_{1}\right) /$ forced vital capacity $(\mathrm{FVC})=1.6 /$ 2.14 and a total lung capacity (TLC) of 3.43 litres. Arterial blood gas tensions on room air were within normal limits.

Bronchoscopic examination revealed extrinsic compression of the right upper lobe airways; mucosal biopsy samples were negative though bronchial washings produced some cytologically suspicious cells. A needle biopsy of the thoracic opacity revealed lipoma.

A presumed diagnosis of massive intrathoracic lipomatosis was made and surgical extirpation planned. The surgical approach was via a median sternotomy, right anterior thoracotomy, and a transverse cervical incision. Because of the potential for massive blood loss and problems with infiltration around the heart and great vessels, facilities for cell-saving and cardiopulmonary bypass were available. At surgery there was an enormous lipomatous mass contained within the mediastinal pleura, protruding posterior to the superior vena cava into

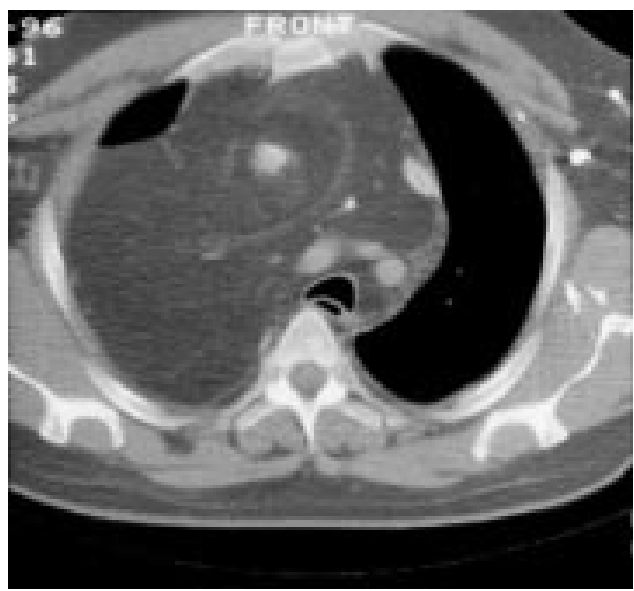

Figure 2 Computed axial tomographic scan showing extensive fatty infiltration of the right hemithorax and infiltration of the mediastinal vessels with airway deviation. 
the right hemithorax, occupying almost half the hemithorax with the phrenic nerve stretched over the mass. The rest of the mediastinum was also infiltrated by diffuse fatty tissue extending into the planes of the neck, both superficially and deeply, and extending to within the pericardium. The fatty mass surrounded and compressed the major veins and pushed the airways posteriorly. Numerous collateral veins over the surface of the lipoma were ligated and divided and the thoracic component of the mass was removed separately. Over $90 \%$ of the mediastinal component was removed with cleaning of major veins, aortic arch and arch vessels. More than $2 \mathrm{~kg}$ of mature fatty tissue was removed. Biopsy specimens of the congenital abnormality at the first costosternal junction showed normal bone and marrow.

\section{Discussion}

Although first described in 1846 by Sir Benjamin Brodie, it was in 1888 that Otto Madelung described a series of patients with benign symmetrical lipomatosis and subsequently the condition received the eponymous term "Madelung's disease". The condition is also known as Launois-Bensaude syndrome following a larger series of patients published by Launois and Bensaude in 1898 . $^{1}$

Classically, the condition presents usually in white middle-aged men of European origin. Patients have a striking appearance with accumulation of excessive amounts of fatty tissue in the neck and upper shoulder girdle. The appearance is that of a "horse collar". The fatty tissue enlarges slowly over several years and patients usually present complaining of the cosmetic deformity. A history of high ethanol intake can often be gained from the patient ${ }^{2}$ but cessation of alcohol does not lead to involution of the masses. ${ }^{1}$ Patients may also present with respiratory difficulties due to laryngeal involvement ${ }^{3}$ or mediastinal compression as in this case. ${ }^{2}$ Other associations include impaired glucose tolerance, hyperlipidaemia, hyperuricaemia, ${ }^{4}$ renal tubular acidosis, and liver enzyme abnormalities. In addition to autonomic neuropathies, peripheral motor and sensory neuropathies have also been described with this condition. ${ }^{2}$ Cases are usually isolated with rarely any hereditary tendency, although $10 \%$ of cases of multiple endocrine neoplasia type I have multiple symmetrical lipomatosis as their only non-endocrine tumour. ${ }^{5}$ Respiratory distress is occasionally caused by laryngeal compression, ${ }^{3}$ infiltration of the false vocal cords, or extensive mediastinal compression ${ }^{2}$ as seen in our case. The condition does not spontaneously involute and surgical treatment is usually required. The fatty tissue removed is benign with only a single case report of malignant transformation to liposarcoma. ${ }^{6}$

Treatment involves surgical removal of lipomatous tissue with the option of liposuction to the external areas of fatty infiltration. ${ }^{7}$ As the lipomatous tissue is diffusely infiltrative, complete removal is not practical. Occasionally the cosmetic and functional benefits of surgical removal are temporary.

The underlying defect is thought to be a primary defect in the lipolytic pathway, possibly as a result of a reduction of the lipoma $\beta$ receptors. Chronic alcohol ingestion is known to reduce the number of peripheral $\beta$ receptors. Treatment with $\beta$ agonists such as salbutamol would appear logical and there is a single report of some success in reducing the lipomatous mass with $12 \mathrm{mg}$ of oral salbutamol in three divided doses. $^{8}$

1 Boozan J, Maves M, Schuller D. Surgical management of benign symmetrical lipomatosis. Laryngoscope 1992;102. benign

2 Enzi G. Multiple symmetrical lipomatosis: an updated clinical report. Medicine (Baltimore) 1984;63:56-64.

3 Moretti JA, Miller D. Laryngeal involvement in benign symmetrical lipomatosis (Madelung's disease). Arch Otolaryngo 1973;97:495-6.

4 Greene M, Glueck C, Fujimoto W, Seegmiller J. Benign symmetrical lipomatosis (Launois-Bensaude adenolipomatosis) with gout and hyperlipoproteinaemia. $A m$ f Med 1970;48:239.

5 Morelli A, Falchetti A, Weinstein L, Fabiani S, Tomassett $\mathrm{P}$, Enzi G, et al. RFLP analysis of human chromosome 11 region q13 in multiple symmetric lipomatosis and multiple endocrine neoplasia type I associated lipomas. Biochen Biophys Res Commun 1995;207:363-8.

6 Tizian C, Berger A, Vykoupil K. Malignant degeneration in Madelung's disease (benign lipomatosis of the neck). $B r f$ Plast Surg 1983;36:187-9.

7 Basse P, Lohmann M, Hovgard C, Alsbjørn B. Multiple symmetric lipomatosis: combined surgical treatment and liposuction. Scand 7 Plast Reconstr Hand Surg 1992;26: $111-2$

8 Leung N, Gaer J, Beggs D, Kark A, Holloway B, Peters T. Multiple symmetrical lipomatosis (Launois-Bensaude syndrome): effect of oral salbutamol. Clin Endocrinol 1987; 7:601-6. 